Buono, Alessandro, Esercito, istituzioni, territorio. Alloggiamenti militari e " case herme » nello Stato di Milano (secoli XVI e XVII)

\title{
Stéphane Perréon
}

\section{(2) OpenEdition \\ 1 Journals}

\section{Édition électronique}

URL : http://journals.openedition.org/abpo/1957

DOI : 10.4000/abpo. 1957

ISSN : 2108-6443

\section{Éditeur}

Presses universitaires de Rennes

\section{Édition imprimée}

Date de publication : 10 avril 2011

Pagination : 197-201

ISBN : 978-2-75351410-2

ISSN : 0399-0826

Référence électronique

Stéphane Perréon, « Buono, Alessandro, Esercito, istituzioni, territorio. Alloggiamenti militari e "case herme » nello Stato di Milano (secoli XVI e XVII)», Annales de Bretagne et des Pays de l'Ouest [En ligne], 118-1 | 2011, mis en ligne le 09 mai 2011, consulté le 21 septembre 2020. URL : http:// journals.openedition.org/abpo/1957 ; DOI : https://doi.org/10.4000/abpo.1957 
une époque très avancée et alerte le chercheur contre l'utilisation sans précaution du réseau communal actuel pour remonter à celui des paroisses de l'Ancien Régime. En outre, il met définitivement un terme à l'hypothèse d'un démembrement croissant du diocèse en de plus en plus petites paroisses. En revanche, le poids des héritages pour expliquer la formation des territoires paroissiaux nous semble un peu sous-évalué par É. Zadora-Rio. Certes, il est sans doute impossible de reconstituer des unités territoriales antérieures au $x^{\mathrm{e}}$ siècle à partir du réseau paroissial, mais on peut penser que des communautés ecclésiales préexistaient aux paroisses et inscrivaient déjà dans l'espace des pratiques cultuelles et sociales. Cet ouvrage très neuf marque un véritable tournant historiographique concernant l'approche de la paroisse et plus largement, la représentation et l'organisation de l'espace au Moyen Âge.

Anne LUNVEN

Buono, Alessandro, Esercito, istituzioni, territorio. Alloggiamenti militari e "case herme " nello Stato di Milano (secoli XVI e XVII), Florence, Firenze University Press, 2009, $354 \mathrm{p}$.

L'histoire militaire de l'époque moderne, quoique souvent circonscrite aux $x V^{e}$ et $\mathrm{XVII}$ siècles, connaît depuis quelques années en Italie un renouveau et un dynamisme indéniables. En témoigne ce livre récent d'A. Buono, jeune chercheur de I'Università Statale de Milan, ouvrage directement issu de sa thèse de doctorat.

S'inscrivant pleinement dans les problématiques actuelles d'une histoire militaire italienne qui se veut également institutionnelle, territoriale et sociale, A. Buono s'interroge sur l'impact de l'armée espagnole dans le duché de Milan aux xvI et $\mathrm{XVII}^{\mathrm{e}}$ siècles. Dans cette optique, le logement militaire, centre de son étude, constitue un " observatoire privilégié » pour appréhender les conséquences de la guerre et de la présence de l'armée sur les institutions locales, leurs finances, leur fonctionnement, ainsi que sur la vie quotidienne des habitants.

L'auteur s'appuie sur des sources variées. En premier lieu, les abondantes archives de l'État de Milan, utilement complétées par de non moins riches fonds communaux (Milan, Pavie et Vigevano pour l'essentiel). Le recours aux archives de la monarchie espagnole conservées à Simancas permet de mieux cerner la politique du pouvoir central. Quant à l'abondante bibliographie, elle offre un panorama de la recherche italienne récente en histoire militaire moderne et, pour d'indispensables mises en perspective, intègre nombre d'études anglo-saxonnes, françaises et espagnoles, sans négliger d'autres sciences humaines (les théories de P. Bourdieu sur le pouvoir inspirent particulièrement l'auteur).

L'étude commence avec le passage de l'État de Milan de la domination des Sforza, continuateurs des Visconti, à celle des Espagnols en 1535, et s'achève dans les années 1650, juste avant la paix des Pyrénées. Sous la domination de Madrid, I'histoire du logement militaire dans le Milanais est faite de constantes expérimentations et d'un certain nombre d'échecs principalement dus au caractère presque incessant des guerres.

Le premier chapitre de l'ouvrage examine la progressive rationalisation du logement des gens de guerre, qui s'accompagne d'une "privatisation » de leur gestion. Les Espagnols ne partent pas de rien : une législation et des administrateurs dévolus aux affaires militaires existaient déjà sous les Sforza et la nouvelle administration se développe sur ce substrat. Après la paix de 1559, 5500 à 6000 soldats stationnent 
en Lombardie. Il s'agit d'abord du très réputé Tercio de Lombardia - un des tercios viejos -, auquel s'ajoute celui de Savoie en 1611, ces soldats d'élite étant pour l'essentiel logés dans les "présides", une vingtaine de places fortes à la frontière de l'État. Viennent ensuite les garnisons ordinaires, infanterie comme cavalerie, puis les troupes " exceptionnelles", passant ou séjournant avant de rejoindre les divers champs de bataille. Toutefois, la position stratégique du Milanais sur la via spagnola - la spanish road de G. Parker - rend cette présence de plus en plus lourde et préjudiciable.

Si une minorité loge dans les places fortes, la plupart des soldats sont accueillis au sein des communautés rurales jusqu'à début du XvII siècle. Le logement en "case de padroni » - l'équivalent du logement dit effectif ou personnel en France - demeure la règle, les habitants devant héberger ces hôtes qui, compte tenu du paiement très aléatoire de leur solde, vivent souvent à leurs crochets. Une évolution notable intervient cependant en 1590, avec la création de l'egualanza generale. Ce système consiste en une péréquation à l'échelle de tout l'État milanais des dépenses liées aux troupes, les cotes d'imposition des particuliers fournissant la base du calcul. Sont théoriquement taxées en premier lieu les communautés n'ayant pas logé de soldats. L'egualanza représente indéniablement un progrès mais $\mathrm{A}$. Buono souligne à juste titre les limites du procédé : lourdeur administrative, corruption, passe-droits. Ainsi, la ville de Milan réussit à ne pas payer sa quote-part entre 1624 et 1627, alors qu'elle représente plus de $25 \%$ du total!

Une autre évolution se fait jour avec la remise en cause, certes progressive et limitée, du privilège des communautés urbaines de ne pas contribuer au logement des gens de guerre. Lorsqu'il faut, en 1610, caser 30000 hommes dans un Milanais devenu une véritable " place d'armes » de la monarchie espagnole, les villes en hébergent forcément. Certaines s'organisent et louent ou achètent des maisons dans ce but, seule Milan parvenant, en fin de compte, à éviter le logement effectif moyennant le versement - irrégulier - d'une contribution pécuniaire. Par ailleurs, dès la fin du xve siècle, l'État se rend compte qu'il ne peut contrôler l'entière gestion de l'armée, si bien qu'il en confie peu à peu des pans entiers à des entrepreneurs privés. C'est d'abord le cas du pain de munition puis du logement, dès les années 1610-1620 : à Côme et Pavie, notamment, I'hébergement des soldats est ainsi dévolu à des entrepreneurs locaux. Ces constatations conduisent $\mathrm{A}$. Buono à critiquer la théorie de la Révolution militaire selon laquelle les innovations techniques, tactiques et financières seraient à l'origine d'un État centralisé : l'exemple lombard montre plutôt un État vite dépassé par l'ampleur des questions militaires et qui s'en remet à des entrepreneurs privés.

Le chapitre suivant, centré sur les années 1640 , s'intéresse à une institution originale : la " commission pour les excès des soldats", créée en 1638. Structure mise à disposition des élites et des corps locaux pour mieux contrôler la machine militaire, la giunta per gli eccessi delle soldatesche est présentée comme la possibilité pour les sujets de recourir directement à la justice du roi, ce que semble confirmer I'interruption des "visites générales » en provenance de Madrid. II faut aussi y voir, néanmoins, un moyen de canaliser le mécontentement face à une pression fiscale croissante. Commission permanente, bien que ne se réunissant que quelques mois par an, la giunta associe les principaux responsables militaires, dont le gouverneur de la province, aux représentants des principaux corps lombards, parfois issus des plus puissantes familles milanaises, comme les Arese.

Recevant de nombreuses plaintes de communautés souvent pressurées mais également désireuses d'obtenir des allégements fiscaux, la commission doit faire face aux difficultés que connaissent alors toutes les armées européennes : officiers déclarant plus de soldats qu'ils n'en ont en réalité ou utilisant des passe-volants 
afin d'obtenir davantage de rations, extorsions d'argent auprès des particuliers, fraudes sur le pain de munition, remontes de cavalerie injustifiées et coûteuses pour la population... Au final, elle peine à contrer ces abus, ayant peu de prises sur les soldats et leurs officiers, dont 20 à $30 \%$ sont d'ailleurs des Lombards. Malgré l'envoi d'ambassades à Madrid afin d'obtenir davantage de pouvoir, la giunta décline progressivement à partir de 1643, en partie paralysée par des désaccords en son sein entre civils et militaires, alors que pèse sur le duché la menace d'une invasion française. Cette commission est finalement abolie en 1654, dans un contexte de victoires et de l'espérance d'une paix prochaine, mais elle renaît, sous une nouvelle forme, en 1661.

L'auteur en vient ensuite, dans le chapitre 3 , au cœur de son sujet, à savoir le logement militaire, plus précisément les premières expérimentations de casernement. Dès la fin du xve siècle, le pouvoir central entend limiter la cohabitation entre civils et militaires, alors que, dès le début du siècle suivant, certaines communautés sollicitent l'autorisation de loger les soldats dans des habitations vides, ce qui confirme que l'impulsion ne vient pas uniquement d'en haut mais naît aussi d'initiatives locales. Le terme de "case herme ", littéralement " maisons inhabitées ", pas construites pour héberger des soldats au départ, s'impose au XVII siècle, A. Buono n'ayant guère trouvé le mot " caserne " avant la fin des années 1660. II émet aussi l'hypothèse que la peste de 1630-1631, en libérant nombre d'habitations, a facilité le mouvement de casernement, qui se généralise dans les années 1640, si bien que le logement personnel est désormais réservé aux situation d'urgence. L'exemption de logement des gens de guerre accordée aux propriétaires louant leurs demeures constitue une autre motivation. Parallèlement, certaines places fortes, comme Alessandria, aménagent de véritables casernes, généralement intégrées ou accolées aux fortifications, alors que de vieilles forteresses ayant perdu leur utilité militaire, comme le château médiéval de Vigevano, hébergent des hommes de guerre moyennant quelques réaménagements.

En matière de casernement, la période 1615-1645 est en fait celle des expérimentations. En 1615, I'ordre du comte de Fuentes, gouverneur du Milanais, de loger les soldats en casa herma entraîne la perplexité voire les doléances de nombreuses communautés rurales, incapables de l'appliquer, notamment parce qu'elles ne peuvent fournir ni l'ameublement ni l'ustensile. Le logement chez l'habitant demeure par conséquent la règle dans les campagnes. Les années 1620 voient une tentative d'organisation provinciale échouer, les communautés ayant le plus grand mal à acquitter l'impôt destiné à l'entrepreneur des case herme du duché. La guerre contre la France, qui débute en 1635, alourdit à nouveau le poids du logement militaire et Philippe IV ordonne la généralisation du casernement à toute la Lombardie. Alors que près de 35000 soldats stationnent dans le duché en 1641, les résistances à cette généralisation du système se multiplient, mues par des motivations fiscales. Les institutions milanaises tardent à répondre, émettent un avis réservé et utilisent des manœuvres dilatoires. Face à la volonté du pouvoir central les Milanais allongent excessivement les délais d'application et misent sur l'inertie, si bien qu'aucune organisation précise n'est encore mise en place en 1645 alors que la Lombardie devient un champ de bataille et subit des dévastations.

Le dernier chapitre de cette étude analyse le fonctionnement des case herme du Duché lors de la décennie 1645-1655, qui voit dans un premier temps l'apogée du système puis sa déliquescence rapide. Le Duché désigne non la totalité de l'État de Milan mais la plus importante de ses neuf provinces, qui concentre à plusieurs reprises plus de la moitié de tous les soldats présents sur le territoire lombard. Alors que le pouvoir madrilène n'intervient qu'en cas de crise, différentes institutions locales et provinciales jouent un rôle fondamental dans l'organisation du logement 
militaire. Ainsi, le Magistrat ordinaire, principal tribunal économique et financier de la Lombardie, décide l'affermage des case herme du Duché à un entrepreneur privé, suivant en cela une tendance lourde de " privatisation » de la logistique militaire commune à la plupart des États européen. La cité de Milan, quant à elle, appuie fortement la généralisation du système, soutien qui est tout sauf désintéressé puisque la ville demeure exempte du logement personnel, au contraire de la plupart des autres communautés : favoriser le casernement revient par conséquent à défendre ce privilège. Le Duché, qui représente les autres communautés, entre parfois en conflit avec Milan à propos de la répartition des charges fiscales mais, comme la cité renfloue plusieurs fois ses caisses, ces crises s'estompent vite. Une entreprise de l'« entretien des case herme » est ainsi mise en place à la fin de l'année 1645 et concerne 25 communautés.

Les difficultés économiques et financières consécutives à la guerre conduisent toutefois certains bourgs inclus dans cette entreprise à réclamer l'abolition du casernement. La "région» de la Gera d'Adda, aux confins du Milanais et de la Vénétie, réagit la première dès 1647 et tente, sans succès, de se soustraire au système commun, ce qui aurait remis en cause tout l'équilibre politique et fiscale de la Lombardie. Même le Magistrat ordinaire se fait le contempteur des case herme l'année suivante, conscient du peu de monnaie en circulation dans le monde rural et de l'endettement de nombreuses communautés. En effet, le bon fonctionnement du système de l'entreprise du casernement dépend étroitement de la rentrée de l'impôt ad hoc, ce qui n'est guère le cas.

Les forts contingents en quartiers en 1648 et 1649 (18000 soldats pendant l'hiver) poussent les gros bourgs marchands et manufacturiers de la plaine lombarde à une véritable "rébellion » contre le casernement. En outre, à l'été 1650 , un nouveau gouverneur autorise finalement la Gera d'Adda " à faire les case herme séparément de celles du Duché ». C'est porter un coup fatal au système, qui se grippe irrémédiablement : le mauvais recouvrement de l'impôt oblige l'entrepreneur à ne plus payer ses fournisseurs, personne n'effectuant plus les indispensables réparations dans les maisons louées, qui se dégradent rapidement. Pour éviter la ruine, l'entrepreneur renonce alors à son bail et le Duché se retrouve, en 1651, à devoir gérer directement le logement. Les succès militaires de 1652 n'offrent qu'un court répit au casernement puisque, l'année suivante, le gouverneur permet aux communautés de choisir comment elles souhaitent loger les troupes. Le logement effectif étant plébiscité, le système des case herme prend fin après les quartiers d'hiver 1654-1655. Cet échec s'explique essentiellement par la conjoncture politique et diplomatique, à savoir "l'énorme pression militaire et fiscale qui s'est abattue pendant ces années de guerre sur les sujets lombards».

Dans une ultime sous-partie, A. Buono examine la répartition des quartiers des troupes à l'échelle de la province milanaise. Sans surprise, la cavalerie se concentre dans les espaces où le fourrage abonde, en particulier la plaine du Pô, alors que I'infanterie stationne dans de gros bourgs ou de quasi-villes n'excédant pas 6000 habitants, les autorités évitant soigneusement une dispersion des soldats à travers la campagne. À l'échelle de ces localités, les casernes, grandes maisons aptes à loger des dizaines de soldats, apparaissent relativement dispersées, ne formant jamais un véritable quartier militaire. Quant à l'entretien de ces demeures, les procès-verbaux dressés en 1652, les seuls conservés, en dressent un tableau bien sombre : chambres sans fenêtre, absence de lits, soldats couchant sur la paille, etc.

Au final, grâce à une analyse très fine des différentes institutions impliquées dans le logement militaire, A. Buono dissèque brillamment les jeux de pouvoir au sein de I'État milanais, comme entre cette périphérie lombarde et le centre espagnol. Se font jour des intérêts communs entre la monarchie habsbourgeoise, qui doit diriger un 
empire mondial, et l'oligarchie lombarde, qui veut tirer profit de sa participation à l'administration d'une partie de cet ensemble. Les expérimentations du milieu du $\mathrm{XVII}^{\mathrm{e}}$ siècle, relativement novatrices comparées à ce qui se passe alors en France par exemple, sont entravées par un contexte militaire et fiscal très défavorable. Elles ne donneront leurs fruits qu'une fois la paix revenue, dans le dernier tiers du xvII siècle et le début du xvIII.

Stéphane PERRÉON

Martin, Philippe et Henryot, Fabienne (dir.), Dom Augustin Calmet. Un itinéraire intellectuel, Paris, éditions Riveneuve, 2008, 428 p.

Ce livre rassemble les Actes du colloque organisé les 18-20 novembre 2007 par le Centre de Recherche Universitaire Lorrain en Histoire, en association avec les villes de Nancy et Senones, à l'occasion du $250^{\circ}$ anniversaire de la mort de dom Augustin Calmet (1672-1757), religieux bénédictin de la Congrégation de Saint- Vanne implantée en Lorraine. Celle-ci accorde une grande place aux études et à l'érudition (théologie, exégèse, histoire...), tout comme la Congrégation de Saint-Maur fondée simultanément en France sur son modèle. II n'y a pas lieu de rappeler la part prise par les mauristes dans I'historiographie bretonne aux lecteurs des ABPO. Ceux-ci ne manqueront pas d'établir des parallèles significatifs avec la situation en Lorraine où se retrouvent les enjeux analogues propres à l'écriture d'une histoire provinciale. L'histoire des provinces, alors en développement, est bien souvent au service d'une cause politique. II faut composer entre les exigences des institutions, celles de la noblesse locale et la nécessité de ne pas déplaire à la couronne. Les religieux ont bien conscience de ces enjeux. Leur désir d'impartialité rencontre des obstacles. Des rapports de forces s'établissent entre les puissants et les érudits. On sait que dom Lobineau, chargé par les États de Bretagne de rédiger une Histoire de la Bretagne ne put réaliser le troisième volume initialement prévu du fait de l'intervention des Rohan. Ces derniers demandent à dom Morice de réviser l'oeuvre de son prédécesseur et d'achever son travail selon leurs volontés ${ }^{1}$. A la même époque, dom Calmet se voit confier en 1719, par le duc Léopold la réalisation d'une histoire de la Lorraine, après l'abandon du projet par un autre religieux. II n'a cependant pas le titre officiel d'historiographe. Son Histoire civile et ecclésiastique de la Lorraine (1728-1729), dont certains passages ont l'heur de déplaire au pouvoir politique doit elle aussi subir des remaniements.

Ces Actes sont matière à d'autres rapprochements significatifs avec les milieux de l'érudition monastique au temps de la " sécularisation des savoirs ». En effet, " ce colloque a été l'occasion de constater un paradoxe : I'indéniable célébrité de l'érudit bénédictin va de pair avec de nombreux préjugés à son endroit, et plus encore de nombreuses zones d'ombres entourant le personnage » [p. 7]. II ne s'agit pas de proposer une biographie classique mais d'étudier le personnage de dom Calmet à partir de cinq angles d'approche: "Dom Calmet en ses miroirs », "Dom Calmet et l'ordre de saint Benoît », "Dom Calmet et les sciences sacrées », " Dom Calmet et I'histoire lorraine», «Dom Calmet et ses lecteurs ».

1. QUÉNIART, Jean, « Les mauristes et l'historiographie bretonne » dans Chroniqueurs et historiens de la Bretagne du Moyen Âge au milieu du xx siècle, Rennes, PUR, coll. « Histoire », 2001, p. 111-123. Dom Lobineau publie son Histoire de Bretagne en 1707. L'ouvrage de dom Morice est publié en 1750, le second tome, réalisé par dom Taillandier, en 1756. 